\title{
3 Adipositas und Niere
}

\author{
Achim Jörres
}

In den letzten beiden Jahrzehnten ist eine weltweite Zunahme der Adipositas sowie der mit ihr verbundenen metabolischen und kardiovaskulären Komplikationen zu verzeichnen. Gleichzeitig steigt die Inzidenz der chronischen Niereninsuffizienz, für deren Entstehung und Progression der Diabetes mellitus und die arterielle Hypertonie bekanntermaßen eine wesentliche Rolle spielen. In den letzten Jahren mehren sich die Hinweise, dass die Adipositas nicht nur als Teil des metabolischen Syndroms für die Entstehung und Progression von Nierenerkrankungen von Bedeutung ist, sondern vielmehr einen eigenständigen Risikofaktor darstellt, der über gewichtsreduzierende Maßnahmen einen Ansatzpunkt für nephroprotektive und/oder therapeutische Interventionen bieten könnte (Eknoyan 2011; Hunley et al. 2010; Ritz et al. 2011). Auch im Stadium der terminalen Niereninsuffizienz, also bei Patienten mit chronischer Dialysebehandlung oder nach Nierentransplantation, ist die Prävalenz der Adipositas ansteigend (Kramer et al. 2006). Überraschenderweise scheinen adipöse Dialysepatienten jedoch kein erhöhtes Mortalitätsrisiko im Vergleich zu nicht-adipösen Dialysepatienten zu haben (Schmidt u. Salahudeen 2007). Manche Untersuchungen beschreiben gar eine „reverse Epidemiologie" dergestalt, dass die Adipositas bei chronischen Dialysepatienten zu einer besseren Prognose führt (Kopple 2005).

\subsection{Adipositas - Risikofaktor für Entstehung und Progression von Nierenerkrankungen?}

Eine mögliche Beziehung zwischen Adipositas einerseits und Albuminurie und Bluthochdruck andererseits wurde bereits im Jahre 1923 durch W.E. Preble berichtet, in dessen Fallserie 40\% der übergewichtigen Patienten eine Proteinurie aufwiesen (Preble 1923). Seither wurde eine Vielzahl von Untersuchungen vorgelegt, die nicht nur die Bedeutung der Adipositas als Risikofaktor für die Entstehung und Progression von Nierenerkrankungen im Rahmen des metabolischen Syndroms bestätigen, sondern darüber hinaus eine eigenständige Rolle der Adipositas (neben Diabetes mellitus und Bluthochdruck) nahelegen. Der genannte Zusammenhang zeigte sich dabei in Untersuchungen an unterschiedlichen Patientenkollektiven sowohl in Europa wie auch in Nordamerika oder Asien.

Wang und Mitarbeiter (Wang et al. 2008) legten unlängst eine Metaanalyse von 22 epidemiologischen Studien aus den Jahren 1980-2006 vor. Verglichen mit Normalgewichtigen (definiert als Body-Mass-Index, BMI 18,5-24,9) hatten Übergewichtige (BMI 25,0-29,9) bereits ein um 40\% erhöhtes Risiko für die Entwicklung einer chronischen Niereninsuffizienz, welches sich nahezu verdoppelte bei Patienten mit Adipositas und BMI > 30,o. Frauen wiesen ein signifikant höheres renales Risiko auf als Männer. Insgesamt zeigte sich in dieser Analyse ein Zusammen- 
hang zwischen Adipositas und Nierenerkrankung bei 24,2\% der Männer und 33,9\% der Frauen in den USA bzw. bei 13,8\% der Männer und 24,9\% der Frauen in Industriestaaten. Schließlich begünstigte die Adipositas ebenfalls die Progression der Niereninsuffizienz.

Eine der größten Einzelstudien untersuchte den Zusammenhang zwischen BMI und der Inzidenz von terminaler Niereninsuffizienz an 320.252 erwachsenen Mitgliedern von Kaiser Permanente of Northern California, einem großen auf Gesundheitsvorsorge spezialisierten Konzern, bei dem mehr als ein Drittel der Bevölkerung im Gebiet der San Francisco Bay krankenversichert ist. Die untersuchten Patienten hatten sich zwischen 1964 und 1985 einem Gesundheitscheck unterzogen, bei dem auch Größe und Gewicht erhoben worden waren. Durch Abgleich mit den Daten des USRDS (U.S. Renal Data System) bis zum Jahre 2000 fanden sich in diesem Kollektiv insgesamt 1.471 Fälle von terminaler Niereninsuffizienz. Mithilfe multivariater statistischer Modelle und unter Einbeziehung einer Vielzahl von Variablen (unter anderem Alter, Geschlecht, ethnische Herkunft, Bildung, Raucherstatus, Herzinfarktanamnese, Serum-Cholesterin, Serum-Kreatinin, Proteinurie und Hämaturie) konnte ein signifikant erhöhtes Risiko zwischen Übergewicht und der Entwicklung einer terminalen Niereninsuffizienz gezeigt werden. Verglichen mit Normalgewichtigen war das adjustierte relative Risiko bei Übergewichtigen bereits um 87\% erhöht und stieg weiter steil an in Abhängigkeit vom BMI. Auch nach weiterer Adjustierung (Ausgangsblutdruckwerte sowie Vorliegen von Diabetes mellitus) blieb der BMI zu Beginn der Untersuchung als unabhängiger Prädiktor für die Entwicklung einer terminalen Niereninsuffizienz bestehen (Hsu et al. 2006).

Die mögliche Assoziation zwischen BMI und Risiko für die Entwicklung einer chronischen Nierenerkrankung (definiert als glomeruläre Filtrationsrate, GFR $<60 \mathrm{ml} /$ min/1,73 $\mathrm{m}^{2}$ Körperoberfläche) wurde untersucht in einer Kohorte von 11.104 ursprünglich gesunden Männern, die an der Physicians' Health Study teilgenommen hatten und 14 Jahre später eine Blutuntersuchung durchführen ließen. Nach im Mittel 14 Jahren hatten 1.377 Studienteilnehmer (12,4\%) eine $\mathrm{GFR}<60 \mathrm{ml} / \mathrm{min} / 1,73 \mathrm{~m}^{2}$. Dabei war ein höherer BMI zu Beginn signifikant assoziiert mit einem erhöhten renalen Risiko. Verglichen mit der niedrigsten Quintile $(\mathrm{BMI}<22,7)$ hatten Studienteilnehmer in der höchsten Quintile (BMI > 26,6) ein um 45\% erhöhtes Risiko, eine chronische Niereninsuffizienz zu entwickeln (Gelber et al. 2005).
In einer Fallkontrollstudie in Schweden konzentrierten sich Ejerblad und Mitarbeiter (Ejerblad et al. 2006) auf Patienten mit einer bereits bestehenden moderaten Niereninsuffizienz und analysierten retrospektiv die assoziierten Risikofaktoren. Aus einer Population von 5,3 Millionen Personen im Alter zwischen 18 und 74 Jahren aus dem Schwedischen Nationalen Bevölkerungsregister identifizierten sie 926 Patienten mit Serum-Kreatinin $>3,4 \mathrm{mg} /$ dl bzw. > $300 \mu \mathrm{mol} / \mathrm{l}$ (Männer) oder $>2,8 \mathrm{mg} / \mathrm{dl} \mathrm{bzw}$. $>250 \mu \mathrm{mol} / \mathrm{l}$ (Frauen), die sie mit 998 Kontrollpersonen verglichen.

\section{In logistischen Regressionsmodellen unter Adjustie- rung für diverse Kofaktoren zeigte sich, dass Über- gewicht (BMI > 25) im Alter von 20 Jahren mit einem dreifach erhöhten Risiko einer chronischen Nieren- insuffizienz einherging. \\ Unabhängig vom Alter waren Adipositas (BMI > 30) \\ bei Männern und morbide Adipositas (BMI > 35) bei Frauen mit einem drei-bis vierfach erhöhten renalen Risiko assoziiert.}

Übergewicht und Adipositas sind oftmals vergesellschaftet mit arterieller Hypertonie. Munkhaugen und Mitarbeiter (Munkhaugen et al. 2009) untersuchten daher die mögliche Interaktion zwischen Blutdruck und Körpergewicht hinsichtlich des Risikos, eine terminale Niereninsuffizienz zu entwickeln oder an einer chronischen Nierenerkrankung zu versterben. Ihre Zielsetzung war letztlich eine bessere Risikostratifizierung bei Patienten mit Prä-Hypertension (definiert als systolischer Blutdruck zwischen 120 und $139 \mathrm{mmHg}$ und diastolischer Blutdruck zwischen 80 und $89 \mathrm{mmHg}$ ). Zu diesem Zweck analysierten sie 74.986 norwegische Patienten, die an der ersten Health Study in Nord-Trøndelag (HUNT I) teilgenommen hatten und über insgesamt 21 Jahre nachverfolgt wurden. In diesem Kollektiv ergab sich eine ausgeprägte Korrelation zwischen erhöhtem BMI und Beginn einer Nierenersatztherapie oder Tod mit chronischer Nierenerkrankung. Interessanterweise hatten Patienten mit Prä-Hypertension und BMI < 30 kein erhöhtes renales Risiko, dieses stieg jedoch erheblich an bei Patienten mit BMI $>30$.

Während die Assoziation von Adipositas und renalem Risiko weitgehend gesichert scheint, ist die mögliche Rolle der Adipositas für die Progression der chronischen Niereninsuffizienz weniger klar. In einer Kohorte bestehend aus 125 nicht-diabetischen Patienten, die seit durchschnittlich 10 Jahren wegen moderater chronischer Niereninsuffizienz am Sheffield 
Kidney Institute, England, behandelt wurden, zeigte sich der Ausgangs-BMI als unabhängiger, signifikanter Prädiktor für einen Abfall der glomerulären Filtrationsrate (Othman et al. 2009). In einer longitudinalen Querschnittsuntersuchung beobachteten Gonçalvez Torres und Mitarbeiter 218 übergewichtige Patienten mit Bluthochdruck in Rio de Janeiro, Brasilien. Ihre wesentliche Fragestellung war, inwieweit sich Veränderungen des BMI über die Zeit auf die Nierenfunktion auswirken. Die Daten von insgesamt 150 Patienten konnten nach im Mittel 5,8 Jahren ausgewertet werden. Am Ende der Nachuntersuchungsphase zeigte sich die Nierenfunktion (GFR) um mehr als $1 \mathrm{ml} / \mathrm{min}$ reduziert je $1 \mathrm{~kg} / \mathrm{m}^{2} \mathrm{An}$ stieg des BMI (Gonçalves Torres et al. 2009). Im Gegensatz hierzu stehen die aktuellen Ergebnisse von Khedr und Mitarbeiter (Khedr et al. 2011). Sie führten eine retrospektive Kohortenstudie an ambulanten nephrologischen Patienten des London Health Sciences Centre, Ontario, Kanada, durch. Insgesamt 214 Patienten mit einer nach der MDRDFormel (Formel der Modification of Diet in Renal Disease-Studie) berechneten GFR (eGFR) $<60 \mathrm{ml} / \mathrm{min} /$ $1,73 \mathrm{~m}^{2}$ wurden über $4,5 \pm 1,8$ Jahre nachbeobachtet. Weder in der univariaten Analyse zeigte sich der BMI statistisch assoziiert mit einer Veränderung der eGFR, noch fand sich mit einem BMI-Grenzwert von 30 ein signifikant schnellerer Abfall der eGFR. In der multivariaten Analyse führten hingegen eine hohe Ausgangs-eGFR, eine Proteinurie und das Vorhandensein einer diabetischen Nephropathie zu einem signifikant schnelleren Rückgang der eGFR.

BMI als Maß von Übergewicht und Adipositas geeignet?
Nicht zuletzt wegen solcher divergierender Befunde wur-
de in jüngerer Zeit diskutiert, ob der BMI tatsächlich als
Maß von Übergewicht und Adipositas in klinischen Studi-
en geeignet ist. Der BMI differenziert nicht zwischen Ge-
wicht der Muskulatur und des Fettgewebes oder zwischen
viszeraler und subkutaner Fettmasse. Bei Individuen, die
zwar den gleichen BMI, aber einen unterschiedlichen Ha-
bitus aufweisen (wie etwa eine unterschiedliche Muskel-
masse), könnte daher die alleinige Anwendung des BMI als
numerische Variable des Übergewichts die Aussagekraft
der Analyse klinischer Endpunkte reduzieren (Janssen et
al. 2004).

Die Waist-to-Hip-Ratio (WHR, Taillen-Hüftumfang) wird daher von einigen Autoren als besseres anthropometrisches Maß vor allem bei Personen mit atypischem Habitus angesehen (Snijder et al. 2006). Tatsächlich fanden Elsayed und Mitarbeiter in einer Analyse der Daten von 13.324 Teilnehmern der ARIC- (Atheroscle- rosis Risk in Communities) und der CHS-Studie (Cardiovascular Health Study), dass die WHR, aber nicht der BMI, mit dem Auftreten einer chronischen Niereninsuffizienz wie auch der Gesamtmortalität korrelierten (Elsayed et al. 2008).

Die Interpretation vieler epidemiologischer Studienergebnisse ist überdies durch den Umstand erschwert, dass die Nierenfunktion (GFR) in der Regel mithilfe einschlägiger Formeln wie der nach Cockcroft und Gault (Cockcroft u. Gault 1976) oder MDRD (Levey et al. 1999) hochgerechnet bzw. abgeschätzt (und entsprechend mitunter als eGFR, estimated GFR angegeben) wurden. Diese Formeln wurden jedoch nicht für adipöse Patienten entwickelt und evaluiert und können um bis zu $10 \mathrm{ml} / \mathrm{min} / 1,73 \mathrm{~m}^{2}$ von der tatsächlichen (gemessenen) GFR abweichen (Friedman et al. 2010). Schließlich kann die Normalisierung der GFR auf 1,73 $\mathrm{m}^{2}$ Körperoberfläche zu einer systematischen Unterschätzung der tatsächlichen GFR bei Übergewichtigen und Adipösen führen, deren Körperoberfläche zumeist 1,73 $\mathrm{m}^{2}$ überschreitet (Wuerzner et al. 2011).

\subsection{Morphologische und funktionelle Veränderungen bei Adipositas-assoziierter Nephropathie}

Erste histologische Studien zur Auswirkung der Adipositas auf die renale Morphologie wurden bereits vor mehr als 50 Jahren durchgeführt. In einer tierexperimentellen Arbeit zeigten Kennedy und Mitarbeiter, dass Überfütterung von Ratten zu einem Anstieg der Nierengröße und der Anzahl von Nierenzellen führt (Kennedy 1957). Nach einigen Monaten Latenz waren die histologischen Läsionen in der Niere vergleichbar mit denen, die bei alten, aber normalgewichtigen, Ratten gefunden wurden. In den 8oer-Jahren des vorigen Jahrhunderts wurde durch Autopsiestudien (Kasiske u Napier 1985) und zunehmend auch durch Nierenbiopsien an Patienten bestätigt, dass die Veränderungen bei adipösen Patienten zwar den klassischen Krankheitsbildern wie einer Glomerulosklerose ähnlich, von diesen aber abgrenzbar sind (Kasiske u. Crosson 1986).

In den letzten Jahren wurde schließlich das Verständnis der Adipositas-assoziierten Clomerulopathie mit Hilfe großer Nierenbiopsieserien weiterentwickelt. Diese ist im Vergleich zu einer idiopathischen fokalen und segmentalen Glomerulosklerose (FSCS) sowohl durch einen milderen klinischen Verlauf mit einer geringeren Inzidenz eines nephrotischen Syndroms wie auch durch besondere histologische Ver- 
änderungen (konsistenter Nachweis einer Clomerulomegalie; mildere Veränderungen der PodozytenFußfortsätze) gekennzeichnet. Die Adipositas-assoziierte Glomerulopathie scheint in jüngerer Zeit allerdings an Häufigkeit zuzunehmen. Kambham und Mitarbeiter fanden an 6.818 nativen Nierenbiopsien aus den Jahren 1986 bis 2000 einen progressiven Anstieg der Inzidenz auf das Zehnfache, nämlich von $0,2 \%$ im Zeitraum 1986-1990 auf 2,0\% in 1996-2000 $(\mathrm{P}<\mathrm{0}, 0001)$ (Kambham et al. 2001). Eine vergleichbare Tendenz berichteten Chen und Mitarbeiter, die 10.093 Biopsate untersuchten, welche zwischen Februar 2002 und November 2006 an der nephrologischen Universitätsklinik Nanjing/China entnommen worden waren. Dabei fanden sie in go Fällen $(0,89 \%)$ eine Adipositas-assoziierte Glomerulopathie, wobei die Frequenz dieser Befunde in den ersten beiden untersuchten Jahren noch bei o, $62 \%$ lag und in den Folgejahren auf durchschnittlich 1,0\% stieg. Insgesamt waren in dieser Serie die Patienten mit Adipositas-assoziierter Glomerulopathie überwiegend jung (37,5 $\pm 9,3$ Jahre) und männlich $(67 \%)$ und hatten einen mittleren BMI von 31,2 $\pm 3,3$ (Chen et al. 2008).

Neben den genannten histomorphologischen Auffälligkeiten führt die Adipositas auch zu funktionellen Veränderungen der Niere. Sowohl bei der genetisch bedingten adipösen Zucker-Ratte wie auch bei überfütterten Hunden lässt sich ein Anstieg des effektiven Plasmaflusses, der Filtrationfraktion und der GFR nachweisen, die mit einer vermehrten Albuminurie einhergehen (Henegar et al. 2001; O'Donnell et al. 1985). Vergleichbare Veränderungen finden sich auch beim Menschen. So untersuchten Chagnac und Mitarbeiter die glomeruläre Funktion bei zwölf ausgeprägt adipösen, nicht-diabetischen Probanden (BMI > 38) und fanden eine um 51\% erhöhte GFR, einen um $31 \%$ erhöhten renalen Plasmafluss (RPF) und eine entsprechend erhöhte Filtrationsfraktion im Vergleich zu neun Kontrollpersonen. Weitere Untersuchungen mittels Infusion von Dextranlösungen unterschiedlicher Molekülgröße wiesen darauf hin, dass den Befunden eine renale Vasodilatation überwiegend im Bereich der afferenten Arteriole zugrunde lag (Chagnac et al. 200o). Eine weitere Entstehungskomponente der erhöhten Filtrationsfraktion ist darüber hinaus vermutlich die Vasokonstriktion der efferenten Arteriolen. Dies legen jedenfalls tierexperimentelle wie auch klinische Befunde nahe, denen zufolge eine Inhibition des Renin-Angiotensin-Systems die Adipositas-assoziierte Albuminurie günstig beeinflusst (Blanco et al. 2005; Praga et al. 1995). Bestätigt wurden diese Befunde in einer aktu- ellen post hoc Analyse der REIN Studie (Ramipril Efficacy in Nephropathy). In dieser Studie untersuchten Mallamaci und Mitarbeiter die Frage, ob Übergewicht und Adipositas den nephroprotektiven Effekt einer ACE-Inhibition mit Ramipril beeinflussen. Von den 337 Studienteilnehmern mit bekanntem BMI waren $105(31,1 \%)$ übergewichtig und $49(14,5 \%)$ adipös. Tatsächlich war bei diesen der nephroprotektive Effekt der ACE-Inhibition ausgeprägter als bei normalgewichtigen Patienten (Mallamaci et al. 2011). Schließlich gibt es Anhaltspunkte dafür, dass es bei der Adipositas zu einem erhöhten sympathischen Vasotonus kommt, der die genannten hämodynamischen Veränderungen mitbeeinflusst (Esler et al. 2001; Hall et al. 2003).

Klinisch imponiert als Folge der glomerulären Hyperfiltration in erster Linie eine Proteinurie. Die Erstbeschreibung der Assoziation einer Adipositasassoziierten Glomerulopathie mit einer Proteinurie stammt von Weisinger und Mitarbeitern, die im Jahre 1974 über vier Patienten mit morbider Adipositas (BMI > 50) berichteten, die ein nephrotisches Syndrom aufwiesen. Keiner dieser Patienten hatte Diabetes mellitus und nur zwei von ihnen einen arteriellen Hypertonus. Interessanterweise gelang in allen vier Fällen durch diätetische Gewichtsreduktion eine Verminderung der Proteinurie (Weisinger et al. 1974). In der Regel manifestiert sich die Adipositasassoziierte Clomerulopathie jedoch zunächst als Albuminurie und nimmt nur selten nephrotisches Ausmaß an (Chen et al. 2008). Dabei zeigt die Proteinurie in manchen Studien eine deutliche und von anderen Variablen unabhängige Assoziation mit dem BMI. So fand sich in einer multivariaten post hoc Analyse von 652 Teilnehmern der AASK-Studie (African American Study of Kidney Disease) ein Anstieg der Proteinurie um 3,5\% und der Albuminurie um 5,6\% je $2 \mathrm{~kg} / \mathrm{m}^{2}$ höherem BMI (Toto et al. 2010). Die Assoziation von BMI und Proteinurie war dabei unabhängig von Geschlecht, Alter, systolischem Blutdruck, Blutglukose, Serum-Kreatinin und -Harnsäure (s. Tab. 1).

\subsection{Eine Rolle für gewichtsreduzierende Interventionen?}

Der in vielen untersuchten Kohorten gefundene $\mathrm{Zu}$ sammenhang zwischen Adipositas und Niereninsuffizienz weckt naturgemäß die Hoffnung, dass durch gewichtsreduzierende Interventionen ein günstiger Effekt auf Proteinurie und Nierenfunktion erzielt werden kann. Systematische Reviews (Afshinnia et 
Tab. 1 Renale Auswirkungen der Adipositas

hämodynamische Veränderungen
effektiver Plasmafluss $\uparrow$
glomeruläre Filtrationsrate $\uparrow$
glomeruläre Filtrationsfraktion $\uparrow$
morphologische Veränderungen
Nierengewicht $\uparrow$
Glomerulomegalie
Glomerulosklerose
Podozytenveränderungen
funktionelle Veränderungen
Albuminurie
Proteinurie
chronische Niereninsuffizienz
terminale (dialysepflichtige) Niereninsuffizienz

al. 2010; Navaneethan et al. 2009) der dreizehn bis zum Jahre 2009 publizierten klinischen Studien an Patienten mit chronischer Niereninsuffizienz ergaben insgesamt einen positiven Effekt von nicht-chirurgischen Maßnahmen zur Gewichtsreduktion auf Proteinurie, Blutdruck und Progression der Niereninsuffizienz. Bei Patienten mit morbider Adipositas konnte mittels bariatrischer Chirurgie eine Normalisierung der GFR sowie eine Reduktion der Mikroalbuminurie erzielt werden. Eine neuere Studie (Shen et al. 2010) untersuchte 63 Patienten mit bioptisch gesicherter Adipositas-assoziierter Nephropathie, die an einem ärztlich überwachten Programm zur Gewichtsreduktion teilnahmen. Nach 6 Monaten zeigten 27 Patienten, die im Mittel 8,3 \pm 4,0\% Gewicht verloren hatten, einen Rückgang der Proteinurie um $35,3 \%$, nach 24 Monaten eine Gewichtsreduktion von $9,2 \pm 3,8 \%$ sowie einen Abfall der Proteinurie um $51,3 \%$. Hingegen zeigten die Patienten, welche an Gewicht zunahmen, auch eine verstärkte Proteinurie.

Insgesamt ist allerdings festzuhalten, dass die bislang vorliegenden Studien zum Effekt von gewichtsreduzierenden Interventionen auf die Nierenfunktion zumeist an kleinen Patientenkollektiven und nur über eine vergleichsweise kurze Beobachtungszeit durchge $\neq$ führt wurden. Es müssen also sicherlich noch weitere prospektive Untersuchungen folgen, um den Wert der verschiedenen Interventionen zweifelsfrei zu belegen. Dies gilt natürlich insbesondere für invasive, chirurgische Prozeduren.

\subsection{Adipositas und terminale Niereninsuffizienz}

Epidemiologische Befunde, erhoben an großen Kollektiven, belegen, dass die Adipositas bei chronischen Dialysepatienten keinen negativen Prognosefaktor darstellt, vielmehr in einer Reihe von Untersuchungen mit einer besseren Überlebenswahrscheinlichkeit verknüpft ist (Kalaitzidis et al. 2011; Kalantar-Zadeh et al. 2005). In der bislang größten Studie analysierten Johansen und Mitarbeiter die Daten von $418.055 \mathrm{~Pa}-$ tienten, die zwischen April 1995 und November 2000 eine chronische Dialysebehandlung begannen (Johansen et al. 2004).

\section{Auch nach Adjustierung unterschiedlicher demo- grafischer, laborchemischer oder Komorbiditätsfak- toren zeigte sich, dass ein hoher BMI, auch im ext- rem erhöhten Bereich, mit einer erhöhten Überle- benswahrscheinlichkeit verbunden war.}

Dies galt für Weiße, Afro-Amerikaner und Hispanos, jedoch nicht für Patienten mit asiatischer Herkunft. Gleichermaßen war ein hoher BMI assoziiert mit einer niedrigeren Hospitalisierungsrate. Diese nordamerikanischen Befunde konnten unlängst von einer Studie aus Südeuropa bestätigt werden, welche prospektiv die Daten von 5.592 inzidenten Dialysepatienten erhob, die zwischen Januar 2000 und September 2005 in einem von 85 Dialysezentren in Portugal, Frankreich oder Italien behandelt wurden. Etwa die Hälfte der Patienten (46,4\%) war normalgewichtig (BMI 20-24,9). Nach einer mittleren Beobachtungsdauer von 2,0 \pm 1,6 Jahren zeigte sich für übergewichtige wie auch adipöse Patienten ein signifikant niedrigeres Sterblichkeitsrisiko (Chazot et al. 2009).

Möglicherweise besitzen auch Veränderungen des „Trockengewichts“ von Dialysepatienten über die Zeit prognostische Relevanz. In einer repräsentativen Kohorte aus 121.762 Patienten, die dreimal wöchentlich Hämodialyse erhielten, erfassten Kalantar-Zadeh und Mitarbeiter (Kalantar-Zadeh et al. 2010) über jeweils 3 Monate gemittelt das Gewicht nach Dialyse sowie das Serum-Kreatinin (als Surrogatparameter für die Muskelmasse). Sowohl hoher BMI wie auch höheres Serum-Kreatinin waren unabhängig und signifikant mit höherer Überlebenswahrscheinlichkeit korreliert, während Gewichtsverlust mit einer höheren Mortalität assoziiert war, es sei denn, es kam gleichzeitig zu einem Anstieg des Serum-Kreatinins. Diese Daten legen nahe, dass sowohl ein höherer BMI mit größerer Muskelmasse wie 
auch ein diskordanter Zuwachs an Muskelmasse bei gleichzeitiger Gewichtsabnahme eine günstigere Prognose bedingen. Hieraus leitet sich die interessante Frage ab, ob muskelaufbauende Interventionen bei chronischen Dialysepatienten möglicherweise zu einer Prognoseverbesserung führen könnten.

Eine Reihe unterschiedlicher Erklärungen steht für das Phänomen der „reversen Epidemiologie“ der Adipositas in der chronischen Niereninsuffizienz zur Diskussion (Kopple 2005). Möglicherweise haben Patienten mit höherem BMI eine bessere Prognose, weil Patienten mit niedrigem BMI eine Protein-Energie-Mangelernährung aufweisen und so einen $\mathrm{Ge}-$ wichtsverlust während Dialyse entwickeln. Ursächlich kommt hier zunächst eine reduzierte Nahrungsaufnahme und Anorexie auf dem Boden von Urämie, Volumenüberladung oder auch großer Mengen oraler Medikamente infrage. Andererseits gibt es Hinweise auf einen verstärkten Katabolismus, der im Rahmen des Malnutritions-Inflammations-Komplexes angesiedelt wird (Kalantar-Zadeh et al. 2003; Stenvinkel et al. 1999). Unlängst wurde gezeigt, dass eine Protein-Energie-Mangelernährung die Assoziation von orexigenen Peptiden, Inflammation und Mortalität von Hämodialysepatienten amplifiziert (Carrero et al. 2011). Nicht zuletzt ist das Fettgewebe eine wesentliche Quelle von Adipokinen und Zytokinen, welche für die Regulation von Entzündungsvorgängen eine wichtige Rolle spielen und die bei adipösen Patienten ein günstigeres, antiinflammatorisches Profil aufweisen könnten (Kalantar-Zadeh et al. 2005). Letztlich bleibt allerdings die Frage offen, ob die Assoziation von Adipositas und reduzierter Mortalität im Dialysekollektiv tatsächlich eine kausale ist oder ob die Adipositas bei Dialysepatienten schlicht als „Marker“ für bessere Gesundheit anzusehen ist und es die kränkeren Patienten sind, die Gewicht verlieren (Schmidt u. Salahudeen 2007).

\section{Fazit für die Praxis}

Die Adipositas ist neben dem Diabetes mellitus und der arteriellen Hypertonie als wichtiger Risikofaktor für die Entwicklung einer chronischen Niereninsuffizienz anzusehen. Bei Patienten mit Anzeichen einer beginnenden Nierenschädigung sollte das Augenmerk darauf gerichtet werden, durch gezielte Schulung und Diätberatung eine Gewichtsreduktion zu erzielen. Diese kann die glomeruläre Hämodynamik verbessern und eine bestehende Albuminurie reduzieren. Daneben ist die Blutdruckeinstellung zu optimieren, wobei in der Regel die Verwendung eines ACE-Inhibitors oder AT1-Blockers zu empfehlen ist. Bei chronischen Dialyse- patienten hingegen ist eine Kalorienreduktion mit dem Ziel einer Gewichtsreduktion auf dem Boden der bisher vorliegenden Erkenntnisse nicht generell empfehlenswert, es sei denn, diese wird durch konkrete Komorbiditäten (wie etwa ein Schlafapnoesyndrom oder Beschwerden im Bewegungsapparat) erforderlich. Auch kann bei starker Adipositas eine Gewichtsreduktion im Rahmen der Transplantationsvorbereitung angezeigt sein. Besondere Aufmerksamkeit verlangt hingegen der Dialysepatient mit niedrigem oder (ungewollt) sinkendem BMI. Bei diesem sind weitere Untersuchungen der Körperzusammensetzung angezeigt, um eventuelle Ursachen eines Verlustes an fettfreier Körpermasse zu erkennen und zu behandeln.

\section{Literatur}

Afshinnia F, Wilt TJ, Duval S, Esmaeili A, Ibrahim HN (2010) Weight loss and proteinuria: systematic review of clinical trials and comparative cohorts. Nephrol Dial Transplant 25, 1173-1183

Blanco S, Bonet I, Lopez D, Casas I, Romero R (2005) ACE inhibitors improve nephrin expression in Zucker rats with glomerulosclerosis. Kidney Int Suppl S. 10-14

Carrero II, Nakashima A, Qureshi AR, Lindholm B, Heimburger 0, Barany P, Stenvinkel P (2011) Protein-energy wasting modifies the association of ghrelin with inflammation, leptin, and mortality in hemodialysis patients. Kidney Int 79, 749-756

Chagnac A, Weinstein T, Korzets A, Ramadan E, Hirsch I, Gafter U (2000) Glomerular hemodynamics in severe obesity. Am I Physiol Renal Physiol 278, F817-F822

Chazot C, Gassia IP, Di BA, Cesare S, Ponce P, Marcelli D (2009) Is there any survival advantage of obesity in Southern European haemodialysis patients? Nephrol Dial Transplant 24, 2871-2876

Chen HM, Li SI, Chen HP, Wang QW, Li LS, Liu ZH (2008) Obesity-related glomerulopathy in China: a case series of 90 patients. Am I Kidney Dis 52, 58-65

Cockcroft DW und Gault MN (1976) Prediction of creatinine clearances from serum creatinine. Nephron 16, 31-41

Ejerblad E, Fored CM, Lindblad P, Fryzek J, McLaughlin JK, Nyren 0 (2006) Obesity and risk for chronic renal failure. I Am Soc Nephrol 17, 1695-1702

Eknoyan G (2011) Obesity and chronic kidney disease. Nefrologia 31, 397-403

Elsayed EF, Sarnak M), Tighiouart H, Griffith JL, Kurth T, Salem DN, Levey $A S$, Weiner DE (2008) Waist-to-hip ratio, body mass index, and subsequent kidney disease and death. Am | Kidney Dis 52, 29-38

Esler M, Rumantir M, Wiesner G, Kaye D, Hastings I, Lambert G (2001) Sympathetic nervous system and insulin resistance: from obesity to diabetes. Am I Hypertens 14, 304S-309S

Friedman AN, Strother M, Quinney SK, Hall S, Perkins SM, Brizendine EJ, Inman M, Gomez G, Shihabi Z, Moe S, Li L (2010) Measuring the glomerular filtration rate in obese individuals without overt kidney disease. Nephron Clin Pract 116, c224-c234

Gelber RP, Kurth T, Kausz AT, Manson JE, Buring JE, Levey AS, Gaziano JM (2005) Association between body mass index and CKD in apparently healthy men. Am / Kidney Dis 46, 871-880 
Goncalves Torres MR, Cardoso LG, de Abreu VG, Sanjuliani AF, Francischetti EA (2009) Temporal relation between body mass index and renal function in individuals with hypertension and excess body weight. Nutrition 25, 914-919

Hall JE, Kuo II, da Silva AA, de Paula RB, Liu J, Tallam L (2003) Obesity-associated hypertension and kidney disease. Curr Opin Nephrol Hypertens 12, 195-200

Henegar JR, Bigler SA, Henegar LK, Tyagi SC, Hall JE (2001) Functional and structural changes in the kidney in the early stages of obesity. I Am Soc Nephrol 12, 1211-1217

Hsu CY, McCulloch CE, Iribarren C, Darbinian J, Go AS (2006) Body mass index and risk for end-stage renal disease. Ann Intern Med 144, 21-28

Hunley TE, Ma LI, Kon V (2010) Scope and mechanisms of obesityrelated renal disease. Curr Opin Nephrol Hypertens 19, 227-234

Janssen I, Katzmarzyk PT, Ross R (2004) Waist circumference and not body mass index explains obesity-related health risk. Am I Clin Nutr 79, 379-384

Johansen KL, Young B, Kaysen GA, Chertow GM (2004) Association of body size with outcomes among patients beginning dialysis. Am I Clin Nutr 80, 324-332

Kalaitzidis RG und Siamopoulos KC (2011) The role of obesity in kidney disease: recent findings and potential mechanisms. Int Urol Nephrol 43, 771-784

Kalantar-Zadeh K, Abbott KC, Salahudeen AK, Kilpatrick RD, Horwich TB (2005) Survival advantages of obesity in dialysis patients. Am / Clin Nutr 81, 543-554

Kalantar-Zadeh K, Ikizler TA, Block G, Avram MM, Kopple JD (2003) Malnutrition-inflammation complex syndrome in dialysis patients: causes and consequences. Am / Kidney Dis 42, 864-881

Kalantar-Zadeh K, Streja E, Kovesdy CP, Oreopoulos A, Noori N, Jing I, Nissenson AR, Krishnan M, Kopple ID, Mehrotra R, Anker SD (2010) The obesity paradox and mortality associated with surrogates of body size and muscle mass in patients receiving hemodialysis. Mayo Clin Proc 85, 991-1001

Kambham N, Markowitz GS, Valeri AM, Lin I, D’Agati VD (2001) Obesity-related glomerulopathy: an emerging epidemic. Kidney Int 59, 1498-1509

Kasiske BL, Crosson JT (1986) Renal disease in patients with massive obesity. Arch Intern Med 146, 1105-1109

Kasiske BL, Napier I (1985) Glomerular sclerosis in patients with massive obesity. Am / Nephrol 5, 45-50

Kennedy $G$ (1957) Effects of old age and over-nutrition on the kidney. Brit Med Bull 13, 67-70

Khedr A, Khedr E, House AA (2011) Body Mass Index and the Risk of Progression of Chronic Kidney Disease. I Ren Nutr 21, 455-461

Kopple ID (2005) The phenomenon of altered risk factor patterns or reverse epidemiology in persons with advanced chronic kidney failure. Am J Clin Nutr 81, 1257-1266

Kramer HJ, Saranathan A, Luke A, Durazo-Arvizu RA, Guichan C, Hou S, Cooper R (2006) Increasing body mass index and obesity in the incident ESRD population. I Am Soc Nephrol 17, 1453-1459

Levey AS, Bosch JP, Lewis JB, Greene T, Rogers N, Roth D (1999) A more accurate method to estimate glomerular filtration rate from serum creatinine: a new prediction equation. Ann Intern Med 130, 461-470

Mallamaci F, Ruggenenti P, Perna A, Leonardis D, Tripepi R, Tripepi G, Remuzzi G, Zoccali C (2011) ACE inhibition is renoprotective among obese patients with proteinuria. I Am Soc Nephrol 22, 1122-1128

Munkhaugen J, Lydersen S, Wideroe TE, Hallan S (2009) Prehypertension, obesity, and risk of kidney disease: 20-year follow-up of the HUNT I study in Norway. Am I Kidney Dis 54, 638-646

Navaneethan SD, Yehnert H, Moustarah F, Schreiber MJ, Schauer PR, Beddhu S (2009) Weight loss interventions in chronic kidney disease: a systematic review and meta-analysis. Clin I Am Soc Nephrol 4, 1565-1574

O’Donnell MP, Kasiske BL, Cleary MP, Keane WF (1985) Effects of genetic obesity on renal structure and function in the Zucker rat. II. Micropuncture studies. J Lab Clin Med 106, 605-610

Othman M, Kawar B, el Nahas AM (2009) Influence of obesity on progression of non-diabetic chronic kidney disease: a retrospective cohort study. Nephron Clin Pract 113, c16-c23

Praga M, Hernandez E, Andres A, Leon M, Ruilope LM, Rodicio IL (1995) Effects of body-weight loss and captopril treatment on proteinuria associated with obesity. Nephron 70, 35-41

Preble WE (1923) Obesity: observations of 1,000 cases. Boston Med Surg | 188, 617-621

Ritz E, Koleganova N, Piecha G (2011) Is there an obesity-metabolic syndrome related glomerulopathy? Curr Opin Nephrol Hypertens $20,44-49$

Schmidt D und Salahudeen A (2007) The obesity-survival paradox in hemodialysis patients: why do overweight hemodialysis patients live longer? Nutr Clin Pract 22, 11-15

Shen WW, Chen HM, Chen H, Xu F, Li LS, Liu ZH (2010) Obesity-related glomerulopathy: body mass index and proteinuria. Clin I Am Soc Nephrol 5, 1401-1409

Snijder MB, van Dam RM, Visser M, Seidell JC (2006) What aspects of body fat are particularly hazardous and how do we measure them? Int | Epidemiol 35, 83-92

Stenvinkel P, Heimburger 0, Paultre F, Diczfalusy U, Wang T, Berglund L, Jogestrand T (1999) Strong association between malnutrition, inflammation, and atherosclerosis in chronic renal failure. Kidney Int 55, 1899-1911

Toto RD, Greene T, Hebert LA, Hiremath L, Lea JP, Lewis JB, Pogue V, Sika M, Wang X (2010) Relationship between body mass index and proteinuria in hypertensive nephrosclerosis: results from the African American Study of Kidney Disease and Hypertension (AASK) cohort. Am I Kidney Dis 56, 896-906

Wang Y, Chen X, Song Y, Caballero B, Cheskin LI (2008) Association between obesity and kidney disease: a systematic review and meta-analysis. Kidney Int 73, 19-33

Weisinger JR, Kempson RL, Eldridge FL, Swenson RS (1974) The nephrotic syndrome: a complication of massive obesity. Ann Intern Med 81, 440-447

Wuerzner G, Bochud M, Giusti V, Burnier M (2011) Measurement of glomerular filtration rate in obese patients: pitfalls and potential consequences on drug therapy. Obes Facts 4, 238-243 


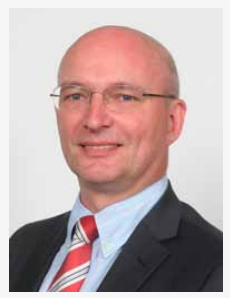

Prof. Dr. med. Achim Jörres

Studium der Medizin an der FU Berlin (1981-1987), danach Wiss. Mitarbeiter der Nephrologie des Univ.-Klin. Rudolf Virchow, Berlin-Charlottenburg. Hier Promotion und Habilitation und Qualifikation als Facharzt für Innere Medizin m.S. Nephrologie und spezielle internistische Intensivmedizin. Im Jahre 2003 Berufung auf die C3-Stiftungsprofessur für Nephrologie und Nierenersatztherapie an der Charité. Seit 2004 Leitender Oberarzt, seit 2009 komm. Klinikdirektor der Medizinischen Klinik m.S. Nephrologie und internistische Intensivmedizin am Campus Virchow-Klinikum der Charité. 